\title{
Risk of Parkinson's disease following gout: a population-based retrospective cohort study in Taiwan
}

\author{
Li-Yu Hu ${ }^{1,2}$, Albert C. Yang ${ }^{1,2}$, Shyh-Chyang Lee ${ }^{3}$, Zi-Hong You ${ }^{4}$, Shih-Jen Tsai ${ }^{1,2}$, Chang-Kuo Hu${ }^{5^{*}}$ and \\ Cheng-Che Shen ${ }^{2,6,7^{*}}$ (D)
}

\begin{abstract}
Background: The progressive neurodegenerative disorder Parkinson disease (PD) is well-established as the second most common neurodegenerative disease. Associations between the sequential risk of PD and gout have been addressed in other studies, but findings have been inconclusive. Accordingly, we executed the present study with the purpose of assessing PD risk in patients with gout.

Methods: From Taiwan's National Health Insurance Research Database, we identified the data of patients newly diagnosed as having gout between January 1, 2000 and December 1, 2000. A cohort of patients without gout, matched for sex and age, was constructed for comparison. Hazard ratios (HRs) and the incidence rate of subsequent PD were calculated for both cohorts and separately for male and female groups. The gout and comparison cohorts consisted of 7900 patients each.
\end{abstract}

Results: The HR for PD was not significantly higher in the gout cohort compared with the control cohort (HR 1.01, 95\% confidence interval $[\mathrm{Cl}], 0.93-1.31, P=.268$ ), even after adjustment for age, urbanization, monthly income, sex, and comorbidities. We did not observe gender differences in the gout-PD association (male: HR 1.01, 95\% Cl, 0.88$1.36, P=.400$; female: HR $1.11,95 \% \mathrm{Cl}, 0.84-1.46, P=.466)$.

Conclusions: Our study identified that there was no protective effect of gout for the risk of PD in the Taiwanese population.

Keywords: Gout, National Health Insurance Research Database, Parkinson disease

\section{Background}

The well-known progressive neurodegenerative disorder Parkinson disease (PD) involves dopaminergic nigrostriatal neuron degeneration, which typically results in motor deficits. It has an estimated worldwide prevalence of 1 to $2 \%$ for individuals over the age of 65 years, rendering it the second most common neurodegenerative

\footnotetext{
*Correspondence: 0312@vghtc.gov.tw; pures1000@yahoo.com.tw ${ }^{5}$ Division of Neurosurgery, Department of surgery, Chiayi Branch, Taichung Veterans General Hospital, No. 600, Sec. 2, Shixian Rd., West District, Chiayi City, Taiwan

${ }^{2}$ School of Medicine, National Yang-Ming University, Taipei, Taiwan Full list of author information is available at the end of the article
}

disease, with the most common being Alzheimer disease [1]. This disorder's global burden has increased by over two times over the past generation because of the increase in the number of elderly individuals [2]. An epidemiology survey has revealed that the PD incidence and prevalence are higher in Western populations than in Asians, including in the Taiwanese people [3, 4]. However, no definite evidence could explain geographic differences until now. There has been considerable progress in determining the environmental and genetic factors that influence the development of PD. Additionally,

(c) The Author(s). 2020 Open Access This article is licensed under a Creative Commons Attribution 4.0 International License, which permits use, sharing, adaptation, distribution and reproduction in any medium or format, as long as you give appropriate credit to the original author(s) and the source, provide a link to the Creative Commons licence, and indicate if changes were made. The images or other third party material in this article are included in the article's Creative Commons licence, unless indicated otherwise in a credit line to the material. If material is not included in the article's Creative Commons licence and your intended use is not permitted by statutory regulation or exceeds the permitted use, you will need to obtain permission directly from the copyright holder. To view a copy of this licence, visit http://creativecommons.org/licenses/by/4.0/ The Creative Commons Public Domain Dedication waiver (http://creativecommons.org/publicdomain/zero/1.0/) applies to the data made available in this article, unless otherwise stated in a credit line to the data. 
accumulating evidence indicates oxidative stress to play a major role in the PD etiology $[5,6]$.

Uric acid (UA) has been demonstrated by previous research to have an antioxidative effect [7-9]. Due to its antioxidant activity, UA was thought to protect neuronal cells, consequently playing a possibly protective role in neurodegenerative diseases. For example, studies have revealed that patients with multiple sclerosis (MS) are known to present lower serum UA levels whereas gout patients have much lower risk for developing MS [10]. Although the role of uric acid in the pathogenesis of PD has not been fully elucidated, there have been several studies demonstrating the associations between the serum UA concentrations [11, 12] and the risk of developing PD or the severity of the progressive symptoms in PD and Alzheimer's disease [13]. Findings reported by previously executed research have been inconsistent with respect to the gout-PD risk association, despite some of such research being large retrospective studies [14-19], as shown in Table 1. For example, an English study found that in the gout group, the increase in the risk of subsequent PD was modest (risk ratio 1.11, 95\% confidence interval [CI], 1.05-1.17) [15]. However, another study in the United Kingdom used a large population-based database and concluded that the risk of PD development is relatively low in individuals having a gout history (risk ratio 0.69, 95\% CI, 0.48-0.99). In addition, other studies have demonstrated variations in PD incidence regarding ethnicity and race $[20,21]$. Asian studies on gout and PD risk are, however, few [18].

To address the previously described inconsistencies in findings regarding the gout-PD risk association and the paucity of Asian studies concerning said association, we executed the present nationwide population-based retrospective cohort study to seek to identify whether an association exists between the two aforementioned illnesses. The following hypothesis constituted the basis for our study: patients with gout would have a relatively low risk of PD.

\section{Methods}

\section{Data sources}

Taiwan's 1995-established National Health Insurance (NHI), covering approximately 99\% of residents of
Taiwan, is a compulsory program covering comprehensive medical care, such as emergency, outpatient, inpatient, and traditional Chinese medicine services [22]. The National Health Insurance Research Database (NHIRD), which is overseen by the National Health Research Institutes, comprises exhaustive information concerning clinic visits, including prescription details as well as diagnostic codes based on the A code and International Classification of Disease, Ninth Revision, Clinical Modification (ICD-9-CM). For this database, data confidentiality is subject to the directives of the NHI Bureau. For executing the present study, we retrieved Longitudinal Health Insurance Database 2000 (LHID2000) data; the LHID2000 comprises the information of 1 million people who were randomly and systematically sampled from the NHIRD. Comparing patients whose information is contained in the NHIRD and those whose information is contained in the LHID2000 demonstrated no significant differences with regard to average distribution of sex or age or the amount of insured payroll [23].

\section{Ethics statement}

The Institutional Review Board (IRB) of Taipei Veterans General Hospital ratified our executed study (IRB number: 2018-07-016 AC). Because the NHIRD comprises secondary data that had been deidentified and maintained for research purposes, written consent from the patients evaluated in this study was unnecessary. Accordingly, the aforementioned IRB provided a formal written waiver of the requirement to obtain patients' written consent.

\section{Study population}

From the LHID2000, we included the data of patients aged more than 20 years and older who received a new diagnosis of gout between January 1, 2000 and December 31, 2000. Gout was defined according to ICD-9-CM code 274 . To ensure patient homogeneity and diagnostic validity, we enrolled patients only if they had at least two consensus diagnoses of gout during the observational period. Patients diagnosed as having PD before enrollment (A code: A221; ICD-9-CM code: 332) were

Table 1 Summary of Studies used to estimate the Risk of Parkinson's Disease among the Patients with Gout

\begin{tabular}{lllll}
\hline Study & Year & Study Design & Ethnic Group (Country) & Risk Ratio (95\% Cl $\mathbf{~})$ \\
\hline Alonso et al. & 2007 & Case-control & Europe (United Kingdom) & $0.69(0.48-0.99)$ \\
De Vera et al. & 2008 & Retrospective cohort & North America (Canada) & $0.70(0.59-0.83)$ \\
Schernhammer et al. & 2013 & Case-control & Europe (Denmark) & $1.06(0.90-1.25)$ \\
Lai et al. & 2014 & Case-control & Asia (Taiwan) & $1.00(0.90-1.11)$ \\
Pakpoor et al. & 2015 & Retrospective cohort & Europe (United Kingdom) & $1.11(1.05-1.17)$ \\
The present study & 2020 & Retrospective cohort & Asia (Taiwan) & $1.01(0.93-1.31)$ \\
\hline
\end{tabular}

${ }^{a} \mathrm{Cl}$ indicates confidence interval 
excluded. Moreover, for each gout patient included in our final cohort, we randomly selected from the LHID2000 the data of a control patient (i.e., a patient not diagnosed as having gout or PD) matched for age, enrollment date, and sex. Observations of all control patients and patients with gout were started since the enrollment date until (1) PD diagnosis by a neurologist, (2) death, or (3) the end date, December 31, 2013. Neurologist-diagnosed PD constituted our primary clinical outcome.

\section{Statistical analysis}

By applying independent $t$ and chi-squared tests, we probed differences between demographic characteristics of patients with gout and control patients. The incidence of newly diagnosed PD in the patient groups was also calculated after stratification of the data by age ( $\geq 65$ or $<$ 65 years), sex, and time since gout diagnosis.

By executing Cox proportional hazards regression, we identified variables predicting PD in the two groups. Many control variables were included in the univariate analysis as covariates; these were sex, age, urbanization, typical comorbidities (diabetes mellitus, chronic liver disease, dyslipidemia, cerebrovascular disease, hypertension, autoimmune disease, nephropathy, and chronic lung disease), and monthly income. We estimated patients' monthly income by using their insurance premiums, typically derived on the basis of the beneficiary's total income. We categorized the estimated monthly income the following groups: no income, low $(<625$ US Dollar), medium (> 625 US Dollar to $<1250$ US Dollar), and high ( $\geq 1250$ US Dollar). In addition, we divided urbanization into the following categories: urban, suburban, and rural. Then, we included univariate analysis factors with a moderate statistically significant relationship (i.e., $P<.1$ ) into a multivariate Cox proportional-hazards regression model by using the forward selection method [24]. Variables predicting PD in male and female groups separately were also identified using the same regression.

For executing data extraction and computation, we employed Perl (Version 5.12.2). Furthermore, we executed data linkage and processing as well as control sampling through Microsoft SQL Server 2005(Microsoft Corp., Redmond, WA, USA). All statistical analysis processes were completed using SPSS (Version 19.0 for Windows; IBM Corp., New York, NY, USA) and SAS (Version 9.2; SAS Institute Inc., Cary, NC, USA). We deemed $P<.05$ as signifying statistical significance.

\section{Results}

\section{Participant selection}

Of the 7900 control individuals and 7900 patients with gout, $83.9 \%$ were determined to be men. We noted the median (interquartile range $[\mathrm{IQR}]$ ) age at the time of enrollment to be 50 (40-64) years; in addition, we observed the median (IQR) follow-up periods for patients with gout and controls to be 13.36 (13.04-13.65) and 13.36 (13.04-13.63) years, respectively. Patients with gout more frequently had comorbidities such as hypertension, cerebrovascular disease, diabetes mellitus, dyslipidemia, nephropathy, autoimmune disease, chronic lung disease, and chronic liver disease than did controls. Clinical and demographic variables of patients with gout and controls are shown in Table 2.

\section{Incidence of PD in gout and control cohorts}

We determined that during the study period, 247 controls (2.63 per 1000 person-years) and 339 patients with gout (3.56 per 1000 person-years) received a PD diagnosis. Between the patients with gout and controls, the PD rate ratio (RR) was $1.36(95 \% \mathrm{CI}, 1.15-1.60, P<.001)$, with the RR remaining higher in patients with gout than in controls after stratification for age ( $\geq 65$ or $<65$ years) and sex (male or female) (Table 3). For stratification by follow-up duration ( $0-1,1-5, \geq 5$ years), the RR of newly diagnosed PD was determined to remain significantly higher in the patients with gout.

\section{Gout and PD risk}

Compared with controls, the gout cohort did not have a significantly higher hazard ratio (HR) for PD development during the follow-up period (HR 1.01, 95\% CI, 0.93-1.31, $P=.268$ ), even after adjustment for sex, age, urbanization, monthly income, and comorbidities (Table 4). After stratification by sex, the HR for PD was not significantly higher among patients with gout than among controls (HR 1.01, 95\% CI, 0.88-1.36, $P=.400$; HR 1.11, 95\% CI, 0.84-1.46, $P=.466$ ) (Supplemental Tables 1 and 2).

\section{Discussion}

Results indicate that the RR of newly diagnosed PD was significantly increased in the gout cohort relative to the control cohort in our study. In this study's gout cohort, the HR for PD was not significantly higher than that in the control cohort. Patients with gout more frequently had hypertension, cerebrovascular disease, diabetes mellitus, nephropathy, dyslipidemia, autoimmune disease, chronic liver disease, and chronic lung disease than did controls. Other previously executed studies have also demonstrated an association between gout and various metabolic and cardiovascular diseases [25-27]. In addition, no gender difference was evident in the gout-subsequent PD risk association.

Results of our study fail to demonstrate an association between gout and PD, even though many studies have determined that UA has an antioxidative effect [7-9]. The possible explanation for this finding is twofold. First, gout is determined to be associated with metabolic and cardiovascular diseases [25, 26]; as demonstrated by 
Table 2 Baseline characteristics of patients with and without gout

\begin{tabular}{|c|c|c|c|c|c|}
\hline \multirow[t]{2}{*}{ Demographic data } & \multicolumn{2}{|l|}{$\begin{array}{l}\text { Patients with Gout } \\
n=7900\end{array}$} & \multicolumn{2}{|c|}{$\begin{array}{l}\text { Patients without Gout } \\
n=7900\end{array}$} & \multirow[t]{2}{*}{$P$ value } \\
\hline & $n$ & $\%$ & $n$ & $\%$ & \\
\hline Age (years) ${ }^{a}$ & $50(40-64)$ & & $50(40-64)$ & & \\
\hline$\geq 65$ & 1791 & 11.5 & 1791 & 11.5 & .999 \\
\hline$<65$ & 6109 & 88.6 & 6109 & 88.6 & \\
\hline \multicolumn{6}{|l|}{ Sex } \\
\hline Male & 5409 & 83.9 & 5409 & 83.9 & .999 \\
\hline Female & 2491 & 16.1 & 2491 & 16.1 & \\
\hline \multicolumn{6}{|l|}{ Comorbidities } \\
\hline Diabetes mellitus & 1664 & 9.6 & 950 & 4.5 & $<.001^{*}$ \\
\hline Hypertension & 3240 & 24.3 & 1887 & 9.8 & $<.001^{*}$ \\
\hline Dyslipidemia & 2230 & 14.0 & 1000 & 4.8 & $<.001^{*}$ \\
\hline Cerebrovascular disease & 1147 & 6.9 & 829 & 3.7 & $<.001^{*}$ \\
\hline Chronic lung disease & 989 & 4.9 & 684 & 1.9 & $<.001^{*}$ \\
\hline Nephropathy & 1219 & 5.9 & 661 & 2.8 & $<.001^{*}$ \\
\hline Chronic liver disease & 2713 & 19.4 & 1638 & 8.7 & $<.001^{*}$ \\
\hline Autoimmune disease & 316 & 2.2 & 163 & 1.2 & $<.001^{*}$ \\
\hline Degree of urbanization & & & & & $<.001^{*}$ \\
\hline Urban & 4186 & 56.9 & 4552 & 62.2 & \\
\hline Suburban & 2853 & 35.2 & 2694 & 32.0 & \\
\hline Rural & 861 & 7.9 & 654 & 5.8 & \\
\hline Income group & & & & & $.023^{*}$ \\
\hline High income & 984 & 15.6 & 1007 & 17.0 & \\
\hline Medium income & 1314 & 49.2 & 1261 & 50.4 & \\
\hline Low income & 4184 & 19.9 & 4073 & 17.8 & \\
\hline No income & 1418 & 15.4 & 1559 & 14.8 & \\
\hline Follow-up years ${ }^{a}$ & 13.36 (13.04-13.65) & & $13.36(13.04-13.63)$ & & $.005^{*}$ \\
\hline
\end{tabular}

${ }^{a}$ indicates Median (interquartile range); ${ }^{*}$ indicates statistical significance

Table 3 Incidence of Parkinson Disease in Patients with and without Gout

\begin{tabular}{|c|c|c|c|c|c|c|}
\hline & \multicolumn{2}{|l|}{ Patients with Gout } & \multicolumn{2}{|l|}{ Patients without Gout } & \multirow{2}{*}{$\begin{array}{l}\text { Rate ratio } \\
(95 \% \mathrm{Cl})\end{array}$} & \multirow[t]{2}{*}{$P$ value } \\
\hline & No. of Parkinson Disease & Per 1000 person-years & No. of Parkinson Disease & Per 1000 person-years & & \\
\hline Total & 339 & 3.56 & 247 & 2.63 & $1.36(1.15-1.60)$ & $<.001^{*}$ \\
\hline \multicolumn{7}{|l|}{ Age } \\
\hline$\geq 65$ & 214 & 12.37 & 159 & 9.31 & $1.33(1.08-1.64)$ & $.006^{*}$ \\
\hline$<65$ & 125 & 1.60 & 88 & 1.14 & $1.40(1.06-1.86)$ & $.015^{*}$ \\
\hline \multicolumn{7}{|l|}{ Sex } \\
\hline Male & 204 & 3.13 & 154 & 2.41 & $1.30(1.05-1.62)$ & $.013^{*}$ \\
\hline Female & 135 & 4.49 & 93 & 3.10 & $1.45(1.11-1.91)$ & $.006^{*}$ \\
\hline \multicolumn{7}{|l|}{ Follow-up } \\
\hline $0-1$ & 33 & 702.13 & 17 & 257.58 & $2.73(1.48-5.22)$ & $<.001^{*}$ \\
\hline $1-5$ & 90 & 62.54 & 65 & 37.06 & $1.69(1.21-2.36)$ & $<.001^{*}$ \\
\hline$\geq 5$ & 216 & 2.30 & 165 & 1.79 & $1.29(1.05-1.59)$ & $.014^{*}$ \\
\hline
\end{tabular}

$\mathrm{Cl}$ indicates confidence interval; ${ }^{*}$ indicates statistical significance 
Table 4 Analyses of risk factors for Parkinson Disease in patients with and without Gout

\begin{tabular}{|c|c|c|c|c|}
\hline \multirow[t]{2}{*}{ Predictive variables } & \multicolumn{2}{|c|}{ Univariate analysis } & \multicolumn{2}{|c|}{ Multivariate analysis } \\
\hline & HR $(95 \% \mathrm{Cl})$ & $P$ value & HR $(95 \% \mathrm{Cl})$ & $P$ value \\
\hline Gout & $1.36(1.15-1.60)$ & $<.001$ & $1.01(0.93-1.31)$ & .268 \\
\hline Age $(<65=0, \geq 65=1)$ & $8.20(6.93-9.71)$ & $<.001$ & $4.41(3.61-5.39)$ & $<.001^{*}$ \\
\hline Sex $($ Male $=0$, Female $=1)$ & $1.37(1.16-1.62)$ & $<.001$ & $0.90(0.76-1.08)$ & .256 \\
\hline \multicolumn{5}{|l|}{ Comorbidities } \\
\hline Diabetes mellitus & $2.83(2.38-3.36)$ & $<.001$ & $1.32(1.08-1.60)$ & $.006^{*}$ \\
\hline Hypertension & $4.62(3.90-5.48)$ & $<.001$ & $1.85(1.52-2.27)$ & $<.001^{*}$ \\
\hline Dyslipidemia & $2.01(1.69-2.38)$ & $<.001$ & $0.90(0.74-1.09)$ & .281 \\
\hline Cerebrovascular disease & $4.63(3.91-5.48)$ & $<.001$ & $2.04(1.69-2.45)$ & $<.001^{*}$ \\
\hline Chronic lung disease & $2.89(2.38-3.52)$ & $<.001$ & $1.25(1.02-1.53)$ & $.035^{*}$ \\
\hline Nephropathy & $2.16(1.77-2.63)$ & $<.001$ & $1.07(0.86-1.32)$ & .563 \\
\hline Chronic liver disease & $1.80(1.53-2.13)$ & $<.001$ & $1.23(1.02-1.47)$ & $.026^{*}$ \\
\hline Autoimmune disease & $1.66(1.13-2.42)$ & .009 & $1.19(0.81-1.75)$ & .369 \\
\hline \multicolumn{5}{|l|}{ Degree of urbanization } \\
\hline Urban & Reference & & Reference & \\
\hline Suburban & $1.34(1.12-1.59)$ & .001 & $1.11(0.92-1.33)$ & .276 \\
\hline Rural & $2.00(1.57-2.54)$ & $<.001$ & $1.32(1.02-1.71)$ & $.033^{*}$ \\
\hline \multicolumn{5}{|l|}{ Income group } \\
\hline High income & Reference & & Reference & \\
\hline Medium income & $5.51(3.59-8.44)$ & $<.001$ & $1.90(1.21-2.99)$ & $.005^{*}$ \\
\hline Low income & $3.94(2.60-5.95)$ & $<.001$ & $0.98(1.04-2.47)$ & $.033^{*}$ \\
\hline No income & $1.30(0.26-2.54)$ & .304 & $1.26(0.76-2.10)$ & .369 \\
\hline
\end{tabular}

$H R$ Indicates hazard ratio, $\mathrm{Cl}$ Indicates confidence interval; * indicates statistical significance

previously executed research, these diseases constitute independent risk factors for PD [28, 29]. For example, a prospective study of the Finnish population revealed that the HR of PD among individuals with type 2 diabetes relative to those without the disease was 1.85 (95\% CI, 1.23-2.80) [30]. The increased prevalence of metabolic diseases may offset the protective effect afforded by UA against the sequential PD risk in patients with gout. The finding of a higher frequency of hypertension, cerebrovascular disease, dyslipidemia, and diabetes mellitus in this study's gout cohort compared with the control cohort supports this explanation. Second, studies have shown an association between gout, a chronic inflammatory disease, and high levels of various inflammatory cytokines, chiefly interleukin (IL)-1, IL-6, and IL-8 [27, 31]. Additionally, although accumulating research evidence suggests the mechanisms of the inflammation in the pathogenesis of PD were far more complicated $[32,33]$ than the assumptions made by previous studies [34, 35], the vast majority of studies still support the idea that inflammation plays a prominent role in mediating the progressive neurodegeneration in PD [36]. Thus, the protective effect of UA in patients with gout may be negated under the influences of the inflammation.
The relationship between gout and PD risk has been evaluated in some retrospective studies, which have yielded inconsistent results [14-19]. A modestly increased risk of subsequent PD in the gout group was observed in one of the studies [15]. However, a decreased risk of PD was found for patients with gout in two other studies [16, 17]. Similar to our study result, two studies failed to identify an association between gout and PD [18, 19]. Different study designs may partially account for the conflicting results. Furthermore, the question as to whether genetic differences along racial or ethnic lines influence PD risk in patients with gout warrants consideration because previously executed research has revealed that race and ethnicity have an influence on the incidence of PD [20,21].

Risk factors for PD have been found to be gender specific [37-39]. For example, one meta-analytic study determined an association between aspirin use by men but not by women with an increased PD risk (men: RR 1.22, 95\% CI, 1.03-1.44; women: RR 0.98, 95\% CI, 0.71-1.37) [38]. With respect to gout and the subsequent risk of $\mathrm{PD}$, one previous study also demonstrated a gender specific difference, finding a decreased PD risk among men with gout (OR 0.60, 95\% CI, 0.40-0.91) but not among women with gout (OR 1.26, 95\% CI, 0.57-2.81) [17]. 
However, in our study, no gender difference was identified regarding the gout-subsequent PD association (Supplemental Tables 1 and 2). Additional studies are required to identify whether the gout-subsequent PD risk association is affected by gender.

Our study has two main strengths: large sample size and specialist-executed PD diagnoses. Moreover, the patient selection process was unbiased. Due to compulsory enrollment in NHI and easy access to low-cost health care, the patient population exhibits low referral biases and high follow-up compliance. However, we must discuss our study's limitations. First, the NHIRD does not have information regarding a family history of PD, environmental factors (such as exposure to herbicides or pesticides), or lifestyle factors (such as coffee or tobacco consumption), but all of these may be associated with PD risk [40-43]. Second, NHIRD-using studies could not obtain records of serum UA levels; accordingly, further research should be performed on whether serum UA levels affect PD risk. Third, this study's follow-up period may have been inadequate for the detection of late-onset PD. Hence, future research must include a longer follow-up period to clarify the long-term risk of PD in patients with gout. Finally, in $\mathrm{NHI}$ claims, the indicated diagnoses are primarily for administrative billing purposes; they are not subject to verification for scientific purposes. Accordingly, the manner in which diagnoses were classified remains unclear in studies using the NHIRD. Thus, we cannot determine the accuracy of the diagnoses.

\section{Conclusion}

In conclusion, no gout-PD association was found in this nationwide retrospective cohort study of patients in the Taiwanese population. We did, however, identify further evidence of associations between gout and metabolic and cardiovascular diseases. Population-based prospective studies in the future should include longer follow-up periods to further probe the gout-PD risk association in different racial or ethnic groups.

\section{Supplementary information}

Supplementary information accompanies this paper at https://doi.org/10. 1186/s12883-020-01916-9.

Additional file 1: Supplemental 1. Analyses of Risk Factors for Parkinson Disease in Male Patients with and withoutGout. Supplemental 2. Analyses of Risk Factors for Parkinson Disease in Female Patients with and withoutGout.

\section{Abbreviations}

PD: Parkinson's disease; UA: Uric acid; Cl: Confidence interval; NHI: National Health Insurance; NHIRD: National Health Insurance Research Database; ICD9-CM: International Classification of Disease, Ninth Revision, Clinical Modification; LHID2000: Longitudinal Health Insurance Database 2000; IRB: Institutional Review Board; IQR: Interquartile range; RR: Rate ratio; HR: Hazard ratio; IL: Interleukin; RR: Relative risk

\section{Acknowledgements}

This manuscript was edited by Wallace Academic Editing.

\section{Authors' contributions}

Study conception and design: S.J.H., C.C.S. and C.K.H. Acquisition of data: A.C.Y., C.C.S. and S.J.T. Analysis and interpretation of data: Z.H.Y., S.J.H. and C.C.S. Draft manuscript: L.Y.H. and S.C.L. All authors had read and approved the final manuscript.

\section{Funding}

This work was supported by grant V108C-038 from the Taipei Veterans General Hospital and grant MOST 108-2314-B-367 -001 - from the Ministry of Science and Technology. The funders had no role in the study design or procedures; in the collection, management, analysis, or interpretation of the data; in the preparation, review, or approval of the manuscript; or in the decision to submit the manuscript for publication.

\section{Availability of data and materials}

The data that support the findings of this study are available from the Taiwan National Health Insurance Research Database (NHIRD). Interested individuals should contact the NHIRD to gain access.

\section{Ethics approval and consent to participate}

The present study was approved by the Institutional Review Board of the Taipei Veterans General Hospital (2018-07-016 AC). No written consent from the study participants was required, because the $\mathrm{NHI}$ data set comprised deidentified secondary data for use in research. The Institutional Review Board of Taipei Veterans General Hospital issued a formal written waiver for the requirement of consent.

\section{Consent for publication}

Not applicable.

\section{Competing interests}

The authors declare no conflict of interest.

\section{Author details}

${ }^{1}$ Department of Psychiatry, Taipei Veterans General Hospital, Taipei, Taiwan. ${ }^{2}$ School of Medicine, National Yang-Ming University, Taipei, Taiwan. ${ }^{3}$ Department of Orthopedics, Chiayi Branch, Taichung Veterans General Hospital, Chiayi, Taiwan. ${ }^{4}$ Division of Nephrology, Department of Internal Medicine, Chiayi Branch, Taichung Veterans General Hospital, Chiayi, Taiwan. ${ }^{5}$ Division of Neurosurgery, Department of surgery, Chiayi Branch, Taichung Veterans General Hospital, No. 600, Sec. 2, Shixian Rd., West District, Chiayi City, Taiwan. ${ }^{6}$ Department of Psychiatry, Chiayi Branch, Taichung Veterans General Hospital, No. 600, Sec. 2, Shixian Rd., West District, Chiayi City, Taiwan. ${ }^{7}$ Center for Innovative Research on Aging Society (CIRAS), National Chung Cheng University, Chiayi, Taiwan.

Received: 27 March 2020 Accepted: 31 August 2020

Published online: 08 September 2020

\section{References}

1. Zhang ZX, Roman GC. Worldwide occurrence of Parkinson's disease: an updated review. Neuroepidemiology. 1993;12(4):195-208.

2. GBD 2016 Parkinson's Disease Collaborators. Global, regional, and national burden of Parkinson's disease, 1990-2016: a systematic analysis for the Global Burden of Disease Study 2016. Lancet Neurol. 2018;17(11):939-53.

3. Muangpaisan W, Mathews A, Hori H, Seidel D. A systematic review of the worldwide prevalence and incidence of Parkinson's disease. J Med Assoc Thail. 2011;94(6):749.

4. Muangpaisan $W$, Hori $H$, Brayne C. Systematic review of the prevalence and incidence of Parkinson's disease in Asia. J Epidemiol. 2009;19(6):281-93.

5. Jomova K, Vondrakova D, Lawson M, Valko M. Metals, oxidative stress and neurodegenerative disorders. Mol Cell Biochem. 2010;345(1-2):91-104.

6. Zhou C, Huang Y, Przedborski S. Oxidative stress in Parkinson's disease: a mechanism of pathogenic and therapeutic significance. Ann N Y Acad Sci. 2008;1147:93-104.

7. Church WH, Ward VL. Uric acid is reduced in the substantia nigra in Parkinson's disease: effect on dopamine oxidation. Brain Res Bull. 1994;33(4): 419-25. 
8. Duan W, Ladenheim B, Cutler RG, Kruman II, Cadet JL, Mattson MP. Dietary folate deficiency and elevated homocysteine levels endanger dopaminergic neurons in models of Parkinson's disease. J Neurochem. 2002;80(1):101-10.

9. Mikami T, Sorimachi M. Uric acid contributes greatly to hepatic antioxidant capacity besides protein. Physiol Res. 2017;66(6):1001-7.

10. Acker T, Acker H. Cellular oxygen sensing need in CNS function: physiological and pathological implications. J Exp Biol. 2004;207(Pt 18): 3171-88.

11. Weisskopf MG, O'Reilly E, Chen H, Schwarzschild MA, Ascherio A. Plasma urate and risk of Parkinson's disease. Am J Epidemiol. 2007;166(5):561-7.

12. Annanmaki T, Muuronen A, Murros K. Low plasma uric acid level in Parkinson's disease. Mov Disord. 2007;22(8):1133-7.

13. Lovell MA, Ehmann WD, Butler SM, Markesbery WR. Elevated thiobarbituric acid-reactive substances and antioxidant enzyme activity in the brain in Alzheimer's disease. Neurology. 1995;45(8):1594-601.

14. Ungprasert P, Srivali N, Thongprayoon C. Gout is not associated with a lower risk of Parkinson's disease: a systematic review and meta-analysis. Parkinsonism Relat Disord. 2015;21(10):1238-42.

15. Pakpoor J, Seminog OO, Ramagopalan SV, Goldacre MJ. Clinical associations between gout and multiple sclerosis, Parkinson's disease and motor neuron disease: record-linkage studies. BMC Neurol. 2015;15:16.

16. De Vera M, Rahman MM, Rankin J, Kopec J, Gao X, Choi H. Gout and the risk of Parkinson's disease: a cohort study. Arthritis Rheum. 2008; 59(11):1549-54

17. Alonso A, Rodriguez LA, Logroscino G, Hernan MA. Gout and risk of Parkinson disease: a prospective study. Neurology. 2007;69(17):1696-700.

18. Lai S-W, Lin C-H, Lin C-L, Liao K-F. Gout and Parkinson's disease in older people: an observation in Taiwan. Int J Gerontol. 2014;8(3):166-7.

19. Schernhammer E, Qiu J, Wermuth L, Lassen CF, Friis S, Ritz B. Gout and the risk of Parkinson's disease in Denmark. Eur J Epidemiol. 2013;28(4):359-60.

20. Van Den Eeden SK, Tanner CM, Bernstein AL, Fross RD, Leimpeter A, Bloch DA, et al. Incidence of Parkinson's disease: variation by age, gender, and race/ethnicity. Am J Epidemiol. 2003;157(11):1015-22.

21. Dahodwala N, Siderowf A, Xie M, Noll E, Stern M, Mandell DS. Racial differences in the diagnosis of Parkinson's disease. Mov Disord. 2009;24(8): 1200-5.

22. Wu CY, Chen YJ, Ho HJ, Hsu YC, Kuo KN, Wu MS, et al. Association between nucleoside analogues and risk of hepatitis B virus-related hepatocellular carcinoma recurrence following liver resection. JAMA. 2012;308(18):1906-14.

23. National Health Insurance Administration, Ministry of Health and Welfare Taiwan: National Health Insurance Annual Report 2014-2015. 2014.

24. Bednarek PH, Creinin MD, Reeves MF, Cwiak C, Espey E, Jensen JT, et al. Immediate versus delayed IUD insertion after uterine aspiration. $\mathrm{N}$ Engl J Med. 2011:364(23):2208-17.

25. Karis E, Crittenden DB, Pillinger MH. Hyperuricemia, gout, and related comorbidities: cause and effect on a two-way street. South Med J. 2014; 107(4):235-41.

26. Abeles AM. Hyperuricemia, gout, and cardiovascular disease: an update. Curr Rheumatol Rep. 2015;17(3):13.

27. Kienhorst LB, van Lochem E, Kievit W, Dalbeth N, Merriman ME, PhippsGreen A, et al. Gout is a chronic inflammatory disease in which high levels of Interleukin-8 (CXCL8), myeloid-related protein 8/myeloid-related protein 14 complex, and an altered proteome are associated with diabetes mellitus and cardiovascular disease. Arthritis Rheumatol. 2015;67(12):3303-13.

28. Zhang P, Tian B. Metabolic syndrome: an important risk factor for Parkinson's disease. Oxidative Med Cell Longev. 2014;2014:729194.

29. Nam GE, Kim SM, Han K, Kim NH, Chung HS, Kim JW, et al. Metabolic syndrome and risk of Parkinson disease: a nationwide cohort study. PLoS Med. 2018;15(8):e1002640.

30. Hu G, Jousilahti P, Bidel S, Antikainen R, Tuomilehto J. Type 2 diabetes and the risk of Parkinson's disease. Diabetes Care. 2007:30(4):842-7.

31. So AK, Martinon F. Inflammation in gout: mechanisms and therapeutic targets. Nat Rev Rheumatol. 2017:13(11):639-47.

32. Yilmaz R, Strafella AP, Bernard A, Schulte C, van den Heuvel L, Schneiderhan-Marra N, et al. Serum inflammatory profile for the discrimination of clinical subtypes in Parkinson's disease. Front Neurol. 2018; 9:1123.

33. Shachar I, Karin NJJ. The dual roles of inflammatory cytokines and chemokines in the regulation of autoimmune diseases and their clinical implications. J Leukoc Biol. 2013;93(1):51-61.
34. Hirsch EC, Hunot SJTLN. Neuroinflammation in Parkinson's disease: a target for neuroprotection? Lancet Neurol. 2009:8(4):382-97.

35. Whitton PS. Inflammation as a causative factor in the aetiology of Parkinson's disease. Br J Pharmacol. 2007:150(8):963-76.

36. Caggiu E, Arru G, Hosseini S, Niegowska M, Sechi G, Zarbo IR, et al. Inflammation, infectious triggers, and Parkinson's disease. Front Neurol. 2019;10:122.

37. Hernan MA, Logroscino G, Garcia Rodriguez LA. Nonsteroidal antiinflammatory drugs and the incidence of Parkinson disease. Neurology. 2006;66(7):1097-9.

38. Gagne JJ, Power MC. Anti-inflammatory drugs and risk of Parkinson disease: a meta-analysis. Neurology. 2010;74(12):995-1002.

39. de Lau LM, Koudstaal PJ, Hofman A, Breteler MM. Serum cholesterol levels and the risk of Parkinson's disease. Am J Epidemiol. 2006;164(10):998-1002.

40. Elbaz A, Moisan F. Update in the epidemiology of Parkinson's disease. Curr Opin Neurol. 2008;21(4):454-60.

41. Costa J, Lunet N, Santos C, Santos J, Vaz-Carneiro A. Caffeine exposure and the risk of Parkinson's disease: a systematic review and meta-analysis of observational studies. J Alzheimer's Dis. 2010;20(Suppl 1):S221-38.

42. Tanner CM, Kamel F, Ross GW, Hoppin JA, Goldman SM, Korell M, et al. Rotenone, paraquat, and Parkinson's disease. Environ Health Perspect. 2011; 119(6):866-72.

43. de Lau LM, Breteler MM. Epidemiology of Parkinson's disease. Lancet Neurol. 2006:5(6):525-35.

\section{Publisher's Note}

Springer Nature remains neutral with regard to jurisdictional claims in published maps and institutional affiliations.
Ready to submit your research? Choose BMC and benefit from:

- fast, convenient online submission

- thorough peer review by experienced researchers in your field

- rapid publication on acceptance

- support for research data, including large and complex data types

- gold Open Access which fosters wider collaboration and increased citations

- maximum visibility for your research: over $100 \mathrm{M}$ website views per year

At BMC, research is always in progress.

Learn more biomedcentral.com/submissions 